\title{
EDITORIAL
}

\section{In the zone: understanding zona reticularis function and its transformation by adrenarche}

\author{
Ian M Bird \\ Perinatal Research Laboratories, Department of Obstetrics and Gynecology, University of Wisconsin-Madison, 7E Meriter Hospital/Park, 202 South Park Street, \\ Madison, Wisconsin 53715, USA \\ (Correspondence should be addressed to I M Bird; Email: imbird@wisc.edu)
}

\begin{abstract}
The human adrenal cortex comprises three distinct zones with unique steroid products, namely the zona glomerulosa, which secretes the mineralocorticoids, the zona fasciculate, which secretes the glucocorticoids and the zona reticularis (ZR), which at adrenarche, begins to produce the so-called adrenal androgens. Of all the adrenal zones, we still understand control of ZR emergence the least, and yet the consequences of such dysregulation can be devastating. Premature adrenarche is a growing problem and the correspondingly inappropriate emergence of ZR function can negatively influence puberty and lead to adult infertility. Our understanding is limited and more needs to be done. The purpose of these three reviews is to provide a survey of where
\end{abstract}

we are in our current understanding of what adrenarche is, and indeed if it is unique to humans at all. Furthermore, these reviews describe what is also known of how the functional ZR emerges during adrenarche and what steroids of physiologic relevance result beyond the widely known DHEA and DHEAS elevated at this time. Such advances in human, primate and indeed stem-cell biology are clearly laying the foundation for new directions in the hunt for the factors involved in the regulation and functional emergence of a ZR at the appropriate time, as well as insight into how they may fail. Given support for these new directions, considerable progress can clearly be made.

Journal of Endocrinology (2012) 214, 109-111
A classic feature of the mammalian adrenal cortex is its distinct histologic zonation. Studies over many decades have further shown that, beyond each zone having a unique function, the centrepetal migration theory says the three zones are in fact formed by a single cell that initiates somewhere in the region of the capsule and migrates to the medullary junction, changing its phenotype as it goes. This of course has echoes of stem-cell biology, and even before pluripotent stem cells were isolated, it was known that transformation of an adrenocortical cell from one zonal phenotype to another is at least in part under endocrine control. Zona glomerulosa $(Z G)$ cells are devoid of any detectable CYP17 (CYP17A1; Fig. 1) and are most responsive to the $\mathrm{Ca}^{2+}$-mobilizing hormones such as angiotensin II (AII) and indeed $\mathrm{K}^{+}$, while the zona fasciculate (ZF) cells are more driven by ACTH and, through associated increases in cAMP, become CYP17 positive. Who wins the battle between ACTH and AII in particular for influence over the cells defines the resulting phenotype, and this can be recapitulated in the H295R cell model (reviewed in Bird et al. (1998)). Nonetheless, the endocrine events that regulate further transformation of the $\mathrm{ZF}$ to a functional zona reticularis $(\mathrm{ZR})$ are also age dependent in vivo and the exact mechanisms are far from clear. A fully functional ZR requires conditions of high CYP17 combined with low HSD3B2 and also CytB5 expression. It is also important to note that while $\mathrm{ACTH}$ is the primary driving force for cortisol production from the ZF and this cortisol in turn delivers clear feedback to the hypothalamus-pituitary to prevent over secretion of $\mathrm{ACTH}$, the $\mathrm{ZR}$ can also respond to ACTH (as well as other factors such as $\mathrm{CRH}$ ) without providing a comparable feedback response given the major ZR products are DHEA and DHEAS. At first glance, the system seems poorly designed, with the clear possibility that over activity or premature activity has no checks and balances in the form of a feedback mechanism. It is thus no surprise that dysfunction can indeed occur and, while not lethal, mistimed early development of ZR capacity to make abundant DHEA can in turn lead to inappropriate and unopposed hyperandrogenism. As the normal compartmentalization of adrenal and gonadal C19 steroidogenesis (Conley \& Bird 1997) breaks down, reproductive disruption can result. Of all the adrenal zones, we still understand control of ZR emergence the least, and 


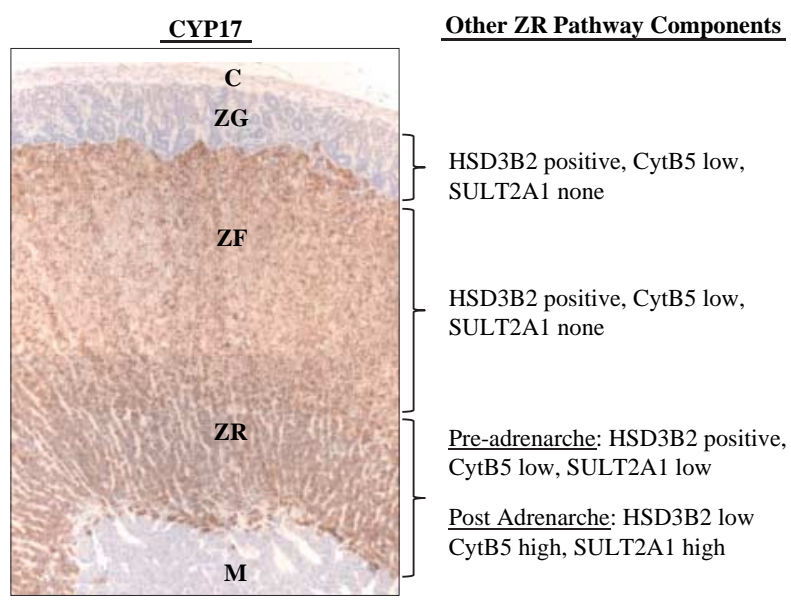

Figure 1 Rhesus adrenal zonation and distribution of ZR-specific steroidogenic enzymes and cofactors known to change in adrenarche. The image shows a cross section of a mature rhesus adrenal stained for CYP17 (Peterson et al. 2001). Staining for CYP17 is indicated by brown color and zones ZG, ZF, and ZR are marked. Width of each zone is also indicated by brackets. $C$ indicates the capsular layer and $\mathrm{M}$ indicates the medulla. Alongside each zone is a further description of the presence or absence of HSD3B2, CytB5, and SULT2A1 in each zone of the adrenal and the pre- and post-adrenarche levels respectively. Alterations in these combined proteins at the time of adrenarche give rise to dramatic increases in the clinical markers DHEA and DHEAS.

still the consequences of such dysregulation are devastating. Clearly more needs to be done. The purpose of these three reviews (Hornsby 2012, Conley et al. 2012, Rege \& Rainey 2012 ) is to provide an up to date survey of where we are in our understanding of how the functional ZR emerges and what steroids of physiologic relevance result from the progression of normal and indeed abnormal adrenarche. The good news is progress in several areas provides a basis for advances on multiple fronts in the near future.

While the overall concept of adrenal centripetal migration and the corresponding need to alter phenotypic function in each zone is readily understandable, the molecular endocrine details remain elusive. There have been many debates that a single 'stem-cell layer' exists and this began with observations of a zona intermedia layer of cells located between the ZG and ZF that does not stain for either zone (neither AT1R positive (ZG) or CYP17 positive (ZF)). Examples of such observations in mammalian species as diverse as the marmoset (Bird \& Pattison 2004) and the sheep (Peterson et al. 2001) are intriguing, but in themselves they do not provide proof of adrenal stem cells. Early studies by Morley et al. (1996) using $\mathrm{B}-\mathrm{Gal}$ reporter under the control of the CYP21 (CYP21A2) promoter in heterozygous mice demonstrated clearly the directional migration of cells of a single lineage from the outside to the inside of the adrenal cortex. Again, however, this does not identify the stem cell itself. The review of Hornsby (2012) presents the case for an adrenal stem-cell lineage and progress made in identification of the factors that may not only transform it through the $\mathrm{ZG}$ and $\mathrm{ZF}$ phenotypes but also the ZR phenotype in humans. This question remains stubborn to dissection; while transplantation of human cells to mice hosts yields steroidogenically functional structures, the transplantation has not yet yielded a completely zonal structure comprising ZG, ZF, and ZR. Likewise, expressions of adrenal-related transcriptional factors in pluripotent stem-cell lines have succeeded in directing steroidogenic pathway expression to some degree but have not yet recapitulated zonal adrenocortical function in vivo. Nonetheless, progress is being made and Hornsby (2012) discusses in detail how further transcription factors such as GATA6 (known to be associated with ZR function) could be prime candidates for targeted expression in these new cell systems.

Beyond humans, there is also longstanding debate on whether adrenarche is also a widespread event that occurs across all primates. In 2012, Hornsby introduces his review with the classic view that, beyond humans, only a limited number of primates also undergo adrenarche. By contrast, Conley et al. (2012) put forward the emerging evidence that the occurrence of adrenarche is far more widespread among nonhuman primates. Indeed, Conley et al. (2012) argue what is variable is not if it occurs, but when it occurs, and the reason this has often been missed is that the period of DHEA/DHEAS 'quiescence' between the regression of the fetal zone and emergence of the functional $\mathrm{ZR}$ is highly variable. In certain cases, the events of fetal zone (FZ) regression and ZR emergence may even be so close as to appear to overlap and so circulating DHEA does not disappear during the functional transition. This is not just a trivial debate of purely academic relevance, it has direct relevance to progression of the field as a whole. Many other research studies in general are undertaken each year in rats and mice that one could argue have little or no relevance to humans, and yet the overwhelming argument to do so often revolves around the time to maturity and so option for rapid developmental and indeed trans-generational studies. Rats and mice lack CYP17 expression completely while many other mammals lack ZR function, and only humans and nonhuman primates express a FZ during development (Conley \& Bird 1997). This gives us few choices for experimental models to study ZR function. If adrenarche is indeed more widespread than initially reported, then not only would comparative studies between primate species be of relevance, but primate models that reach a functional $\mathrm{ZR}$ in $<1$ year and maturity by 18 months offer further options for controlled trans-generational study of endocrine disruptor effects in utero and during early life that would not be possible in humans. The argument by Conley et al. (2012) that adrenarche occurs in a wider list of nonhuman and human primates than has previously been acknowledged is thus not only compelling but also of practical significance. Indeed, since the discovery of CytB5 as a necessary cofactor for high DHEA output that can also be used as a reliable marker for the functional ZR, such studies are now practical as altered steroid products can be mapped against changes in adrenal 
histology even when FZ regression and ZR emergence overlap. Already studies along these lines have suggested that adrenarche may be not only a highly variable but also a tightly regulated process, and while rhesus may initiate adrenarche even before FZ regression is complete (reviewed in Conley et al. (2012)), marmosets may not automatically undergo adrenarche at all in males, or may only undergo a pseudo reversible 'adrenarche' in females in response to social cues and associated changes in ovarian function (Pattison et al. 2009). A comparison of these events and the endocrine and indeed epigenetic processes that regulate them would be highly informative. The review of Rege \& Rainey (this volume) also covers candidate hormones that may be potential regulators of $\mathrm{ZR}$ emergence in humans, and comparative studies of this endocrine system emergence across these diverse primate species and its relationship to ZR emergence and corresponding steroid output may be the best way to confirm or refute their importance in each case before moving to human studies once more.

As our understanding of the molecular endocrine mechanisms behind ZR emergence develops, the question arises whether our clinical definition of adrenarche remains optimal. Clinically, the basis for detection still lies at the level of the major ZR-derived blood-borne steroids. As markers, they are certainly easy to collect and sufficient to indicate altered ZR function, but that is all they indicate. For as long as the more detailed process behind and indeed purpose of adrenarche remain poorly understood, a more precise clinical measure of the biologically active steroid itself remains elusive. To address which of the ZR steroid products are actually biologically active, we must first know what they are. To that end, Rege \& Rainey (this volume) further review our knowledge of the steroid metabolome of adrenarche. While the major steroids by mass (DHEA and DHEAS) are commonly measured for diagnostic purposes, they are inert as androgens and serve instead as substrates for testosterone or estrogen secondary metabolites. Androstenedione is also known to be an additional product of the ZR to a limited extent consistent with the report that HSD3B2 is reduced relative to CYP17, but not absent from the ZR. Combined studies of the full steroid metabolome as a whole both preand post-adrenarche further reveal a more complex picture. In addition to changes in steroid pathway intermediates reaffirming the central importance of changes in CYP17, HSD3B2, and CytB5, as well as SULT2A1 and its cofactors, such analysis also reveals the possible importance of further metabolites such as 11BOHA4. The relevance of this steroid metabolite is its ability to then be metabolized further via HSD17B to $11 \mathrm{OHT} 4$, providing an alternate pathway to the bioactive androgens beyond A4 conversion to $\mathrm{T} 4$ by HSD17B. The emergence of these pathways in the adrenal in adrenarche and indeed puberty are clearly in need of further study, and such knowledge may well provide more evidence for us to reconsider and even redefine adrenarche in a manner that emphasizes more accurately what it is and provides a basis for more clinically relevant diagnosis when it may have failed. The field is clearly set for such translational studies to be undertaken, and it now only requires recognition in the form of funding.

\section{Declaration of interest}

The author declares that there is no conflict of interest that could be perceived as prejudicing the impartiality of the research reported.

\section{Funding}

This research did not receive any specific grant from any funding agency in the public, commercial, or not-for-profit sector.

\section{References}

Bird IM \& Pattison JC 2004 Expression of AT1-R in marmoset whole adrenal glands and adrenocortical cells in culture. Endocrine Research 30 753-757. (doi:10.1081/ERC-200044025)

Bird IM, Mason JI \& Rainey WE 1998 Battle of the kinases: integration of adrenal responses to cAMP, DG and $\mathrm{Ca}^{2+}$ at the level of steroidogenic cytochromes P450 and 3 3 HSD expression in H295R cells. Endocrine Research 24 345-354. (doi:10.3109/07435809809032614)

Conley AJ \& Bird IM 1997 The role of cytochrome P450 17 $\alpha$-hydroxylase and $3 \beta$ hydroxysteroid dehydrogenase in the integration of adrenal and gonadal steroidogenesis via the $\Delta 5$ and $\Delta 4$ pathways. Biology of Reproduction 56 789-799. (doi:10.1095/biolreprod56.4.789)

Conley AJ, Bernstein RM \& Nguyen AD 2012 Adrenarche in nonhuman primates: the evidence for it and the need to redefine it. Journal of Endocrinology 214 121-131. (doi:10.1530/JOE-11-0457)

Hornsby PJ 2012 Adrenarche: a cell biological perspective. Journal of Endocrinology 214 113-119. (doi:10.1530/JOE-12-0022)

Morley SD, Viard I, Chung BC, Ikeda Y, Parker KL \& Mullins JJ 1996 Variegated expression of a mouse steroid 21-hydroxylase/ $\beta$-galactosidase transgene suggests centripetal migration of adrenocortical cells. Molecular Endocrinology 10 585-598. (doi:10.1210/me.10.5.585)

Pattison JC, Abbott DH, Saltzman W, Conley AJ \& Bird IM 2009 Plasticity of the zona reticularis in the adult marmoset adrenal cortex: voyages of discovery in the new world. Journal of Endocrinology 203 313-326. (doi:10.1677/JOE-08-0554)

Peterson JK, Moran F, Conley AJ \& Bird IM 2001 Zonal expression of eNOS in sheep and rhesus adrenal cortex. Endocrinology 142 5351-5363. (doi:10.1210/en.142.12.5351)

Rege J \& Rainey WE 2012 The steroid metabolome of adrenarche. Journal of Endocrinology 214 133-143. (doi:10.1530/JOE-12-0183)

Received in final form 8 June 2012

Accepted 14 June 2012

Made available online as an Accepted Preprint

14 June 2012 